\title{
Management in Bulgarien - einen Blick vor Ort
}

\author{
Z. Kumanov
}

Unter allen Faktoren der gesellschaftlichen Entwicklung - wirtschaftlichen, politischen, sozialen, rechtlichen u.a. - spielt das Management der Prozesse eine effizienzbringende Rolle. Ohne adäquates Management, d.h. Organisation, Führung, strategische Planung und Zielsetzung, gibt es weder Transformation noch Wende. Das gilt sowohl für den Staat oder irgendeinen Wirtschaftszweig, als auch für die Welt einer kleinen oder größeren Firma. Daß das richtige Managen Ordnung schafft, Rahmen und Bedingungen stellt, ist kaum zu bestreiten. In diesem Sinne bildet das Management in Bulgarien keine Ausnahme.

Das Bild vor Ort läßt sich kurz dreidimensional darstellen - die Vergangenheit, die aktuelle Lage und zukünftige Erwartungen. Sicherlich ist so eine Betrachtungsweise schematisch und läßt keine tiefgreifende Analyse der verschiedensten Managementaspekte zu. Zum ersten Nachdenken scheint es uns aber geeignet.

Es gab eine Entwicklungsphase in Bulgarien, etwa bis zum Jahr 1989, in der die Begriffe Planung, Prognose, Zielsetzung, wissenschaftliche Organisation, Arbeitswissenschaften und wissenschaftliche Führung keine leere Inhalte ausdrückten, sondern als Instrumente der Gesamtentwicklung angewandt wurden. Natürlich nannte keiner die Führung eines Betriebes, einer Großfirma, eines Ministerium oder eines Wirtschaftszweig modernes Management. In der Substanz aber wurden die Parameter der Organisation und der Führung im Sinne des Managements angewendet.

Für Bulgarien gab es dabei auch keine Tabus weil das Land mit etwa 4 Mio. Touristen im Jahr, der BRD als zweitgrößten Handelspartner, mit riesiger und konkurrenzfähiger Elektronik-, Hebe-, Transport- und Landwirtschaftlicher Großproduktionen offen für den Westen war. Im gegenseitigen Austausch kamen Technologien und Produkte sowie auch relativ neue Führungskonzepte in die Betriebe. Die Ausbildung war am wissenschaftlichen Stand der Arbeitsorganisation ausgerichtet. Qualifizierung, Requalifizierung, Schulungen der Manager, entlang der ganzen Kette eines Produktes oder sozialer Tätigkeit, wurden zur Regel.

Schlecht oder gut, effizient oder Imitation; man praktizierte auf allen Ebene des wirtschaftlichen- und sozialen Lebens in Bulgarien das Management. Die Ursachen für den Fall des Systems und die Wende sollten daher nicht unbedingt 
im Feld des Managements gesucht werden. Sie sind vielmehr woanders zu finden: sowohl in der gesamten Wirtschaft aber auch im Versagen des politischen Systems.

Nun schreiben wir das Jahr 1998. Die Lage im Bereich des Managements als Element der Transformation zur Marktwirtschaft ist mehr als besorgniserregend. Alles das was gewesen ist, existiert nicht mehr. Keine Führung, keine Organisation im alten Stil, aber auch keine neue Managemententwicklung. Zehn lange Jahre läuft bereits die Umstellung der Wirtschaft und der Gesellschaft. Heute haben wir eine, immer noch schleppende Privatisierung. Die Großbetriebe in den tragenden Industrien - Chemie, Elektronik, Metallurgie, Tourismus sind entweder in ausländischen Händen oder kaputt. In den vormaligen Staatsbetrieben und Firmen wurden regelmäßig die führende Manager gewechselt. So sind allein im letzten Jahr z.B. 3000 der Spitzenvorstände durch neue, im Prinzip ahnungslose Leute ersetzt worden. Es gibt sogar lächerliche Beispiele des Managements, daß z.B. eine Firma, Behörde oder sogar ein ganzer Wirtschaftszweig von Spitzenleuten gemanagt wird, die nie etwas über Führung, Organisation oder gar vom modernen Management gehört haben. Deshalb ist der Transformationsproze $\beta$ sehr schmerzlich. Gerade wegen dem Fehlen an Strategie, Umstrukturierung und notwendigen Investitionen, gepaart mit breiter Korruption, kann man von ernsthafter Managementwicklung kaum reden.

Die neu entstehenden, vorwiegend relativ kleinen Unternehmen, versuchen natürlich, die Offenheit zu Europa zu nutzen und wenden Elemente des Managements sowie des Marketings an. Dazu helfen einige der EU Programme, wie z. B. das Transformprogramm der BRD, das auf die Entwicklung und den Aufbau von kleinen und mittleren Firmen zielt.

Es kommen alle möglichen Berater ins Land. Schwer ist es aber, eigene Initiative zu entwickeln und die Erkenntnisse westlichen Managements voll zu nutzen. Einmal weil Bulgarien unter der Aufsicht des IWF lebt und sich stabilisiert, d.h. der Makro- und Mikrorahmen ist vorgegeben und weiterhin "hinkt" das moderne Management hinter her, weil bei uns immer noch keine wirtschaftliche Freiheit vorhanden ist. Der Staat mischt sich überall im Leben eines Unternehmens ein: etwa 130 Gesetze und Verordnungen schreiben vor, wo und was geschehen muß oder es wird reglementiert, woher und wofür man Genehmigungen zu holen hat. Auch die Angleichung der etwa 2000 Gesetze oder Normen an das EU Rechtssystem läuft im Schneckentempo. Der Zeitfaktor, der auch im Management große Rolle spielt, indiziert kein besonders erfreuliches Bild. Selbstverständlich wird sich die Lage, weil Bulgarien bereits assoziiertes Mitglied der EU ist, ändern müssen. Gerade in den zu erwartenden Änderungen liegen die Aussichten für eine bessere, europäische Managemententwicklung.

Höchstwahrscheinlich soll in den nächsten zwei, drei Jahren die Privatisierung und die Umstrukturierung vollständig beendet sein. Die Anpassung, d.h. die 
Einführung der rechtlichen EU-Normen wird weiter folgen. Den Menschen, den Managern aller Kategorien und Ebenen muß klar gemacht werden, daß ohne moderne Führungsmethoden und Organisation, kein funktionsfähiger Wirtschaftszweig, Firma oder Betrieb hergestellt werden kann und keine Integration in die europäischen Strukturen erfolgen kann.

Aber der Kluft zwischen Managementtheorie und Praxis wird, so hoffen wir einmal überbrückt werden.

Es ist auch zu erwarten, daß das Potential, das bereits vorhanden war, nicht weiter verloren gehen soll, oder auswandert, wie allein etwa die 500000 jungen Leute, die ins Ausland gingen.

Ohne Zweifel sind noch bestimmte Vorurteile, kulturelle Unterschiede, alte Managementvorstellungen $\mathrm{zu}$ überwinden: Man muß lernen, nicht nur technologisch zu denken, sondern auch im Stande sein, die Transformationsprozesse mit neuem Management, d.h. z.B. Leitungsinstrumenten, zu beherrschen. $\mathrm{Ob}$ die Rahmenbedingungen dazu reifen werden, bleibt abzuwarten. Sicher ist nur, daß das Management gemanagt werden sollte. Und zwar vor Ort, im Lande und im Einklang und Mitwirkung der Bulgarien freundlichen EU Staaten. 


\section{Journal for East European Management Studies}

Vol. 3, 1998

\section{Articles}

Page

Ainamo, Antti / Cardwell, William: After Privatisation: Economic Development, Social Transformation and Corporate Governance in the Baltic States

Antal-Mokos, Zoltán: Squeeze play: a case from the Hungarian privatisation

Bennet, Roger: First insights into the transfer of marketing know-how from Western firms to enterprises in Central and East Europe: A qualitative study

Bobek, Samo / Lynn, Monty L. / Lytle, Richard S.: Analysis of Critical Service Factors in two Slowenian Banks Compared to the USA

Clark, Ed: Czech enterprises and the multidivisional form: Legitimation, legacies and structural choice

Cox, Tony / Hooley, Graham / Fahy, John / Beracs, József / Fonfara, Krzysztof / Snoj, Boris: Privatisation Method Effects on Performance and Market Orientation of Central/Eastern European Companies

Gaisbauer, Helmut / Springer, Reiner: Geschäftstätigkeit oberösterreichischer Unternehmen in Zentral- und Osteuropa Ergebnisse einer Befragung von Export-Unternehmen

Gortschewa, Tanja: Internationale Strategien der sich im

Transformationsprozeß befindlichen ost- und mitteleuropäischen Länder

Kelemen, Mihaela / Hristov, Latchezar: From Centrally Planned Culture to Entrepreneurial Culture: The Example of Bulgarian and Romanian Organisations

Matthews, Robin / Yeghaizarian, Ara: Russian Management Style: A Game Theory Approach to Evaluating Decision Making

Savitt, Ronald: Evolving Management Practices in the Czech Republic:

Restructuring and Market Orientation

Shaukat, Ali / Mirza, Hafiz: Country-Specific Investment Motivations in Central Europe: Evidence from British Firms 
Suutari, Vesa: Problems Faced by Western Expatriate Managers in

Eastern Europe: Evidence provided by Finnish Expatriates in Russia and Estonia

Thomson, J. Neil / McNamara, Peter: Two-way learning in west/east mergers $\&$ acquisitions: Short term and long term viewpoints

Zalar, Boštjan: The transformation of companies from the standpoint of participation rights: The case of Slovenia

\section{Forum}

Bakacsi, Gyula: Interview on "Acquisitions and organisational change"

Edwards, Vincent: Researching management in Central and Eastern

Europe: Researcher, manager and management practice.

Grancelli, Bruno: Slovenian firms after privatisation: is there a transformational leadership?

Kovac, Jure: Open Issues at the Conclusion of Privatization (Corporate Ownership Restructuring) in Slovenia

Kovács, András: Interview on "Mergers and acquisitions in the Hungarian sugar industrie"

Pucko, Danijel: Comment and Views on "Open Issues at the Conclusion of Privatisation"

Pucko, Danijel: Comment and views on Researching management in Central and Eastern Europe

Strakhova, Olga A. / Frolov, Denis Y.: Commentary on "Researching management in Central and Eastern Europe

\section{Book Reviews}

Barz, Svetlana Anuschka: Rechnungslegung und Buchführung in Rußland. Jahresabschluß - Buchführungssystem - Normen der Rechnungslegung. Ein Leitfaden mit Buchungsbeispielen (Klaus Müller)

Brezinsky, Horst / Fritsch, Michael (Hrsg.): The Economic Impact of

New Firms in Post-Socialist Countries.

Brezinsky, Horst / Fritsch, Michael (Hrsg.): The Emergence and

Evolution of Markets (John Erpenbeck)

Groenewald, Horst / Leblanc, Bruno (Hrsg.): Personalarbeit auf 
Marktwirtschaftskurs (Uta Wilkens)

Heyse, Volker / Erpenbeck, John: Der Sprung über die

Kompetenzbarriere - Kommunikation, selbstorganisiertes Lernen und

Kompetenzentwicklung von und in Unternehmen (Frank Rudolf)

Remišová, Anna: Podnikatel'ská Etika. Úvod do problematiky. (First

Slovak manual for teaching business ethics) (Martin Lačný)

Schmidt-Tophoff, Johannes: Projektmanagement zur Privatisierung der ostdeutschen Großchemie (Klaus Tragsdorf)

Viola, Mariano R.: Reputationsbildung und Auslandskapital:

Entwicklung der Transformationsländer Ungarn und Polen

(László Lázár)

Warken, Heribert: Unternehmenskontrolle und Finanzmärkte im Transformationsprozeß Osteuropas aus Sicht der Principal-AgentTheorie. (Winfried Liedtke) 